\title{
Politique
}

\section{Répertoire des thèses et mémoires en science politique}

Numéro 7, hiver 1985

Projection internationale du Québec

URI : https://id.erudit.org/iderudit/040494ar

DOI : https://doi.org/10.7202/040494ar

Aller au sommaire du numéro

Éditeur(s)

Société québécoise de science politique

ISSN

0711-608X (imprimé)

1918-6584 (numérique)

Découvrir la revue

Citer ce document

(1985). Répertoire des thèses et mémoires en science politique. Politique, (7),

177-181. https://doi.org/10.7202/040494ar d'utilisation que vous pouvez consulter en ligne.

https://apropos.erudit.org/fr/usagers/politique-dutilisation/ 


\section{Répertoire des thèses et mémoires en science politique}

\section{NOM DE L'AUTEUR-E}

Abbassi, Lahssen

Aouahchi, M. Hammed

Bourque, Marie

Brouillet, Claude

Bruzzesi, Giulio

Castonguay, Jean

Dalpé, Robert

Després, Olivier

Dussault, Georges

Fillion, Yves .

Gagnon, Jacques

Girard, Rémi

Morin, Pierre

Beauregard, Chantal Caron, Michel C.

\section{TITRE}

"Petite bourgeoisie, partis et idéologie socialistes au Maroc" "L'impact des groupes externes et internes sur la prise de décision au Maroc, de 1963 à 1977"

«Révolution, projet révolutionnaire et éducation: les cas de l'Algérie, de Cuba et de la Guinée»

"La C.I.A. et la politique extérieure des États-Unis"

«La corruption et la spéculation comme facteurs de maintien du système politique soviétique»

"Maîtrise sans mémoire"

"Transferts et développement d'une capacité technologique: le cas de Bombardier"

«Réforme constitutionnelle et intégration régionale au Canada"

«La problématique des phénomènes migratoires en Israël: perspective juive"

"La commission canadienne des transports: le transport aérien régional"

"Le système politique urbain, la décentralisation et la planification régionale: une analyse de la loi sur l'aménagement et l'urbanisme"

"Maîtrise sans mémoire"

«Les politiques québécoises relatives à la santé et à la sécurité au travail: les facteurs qui sous-tendent et orientent l'intervention de l'État"

"La diplomatie culturelle du Canada de 1970 à 1980"

"Analyse des notions de société civile et d'État chez Hegel»

\section{UNIVERSITÉ}

Université de Montréal$$
\text { " " }
$$

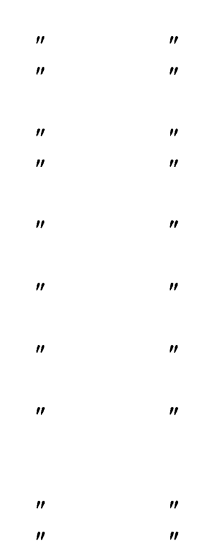

Université d'Ottawa " 
Forget, Denis

Papanikolatos, Nausika (Nancy)

Rainville, Luc-J.

Bélanger, Luc

Bernoussi, Mohamed

Beylerian, Onnig

Biron, Jean-Marc

Boisclair, Jeanne

Gagnon, Nathalie

Gueye, N'Diaga

Johnson, Lynda

Labelle, Gilles

N'Gbiegba-Kognabade, Crépin

Préjean, Marc

Thuot, Jean-François

Vaillancourt, Yves
"La pensée sociale de l'église dans les brochures de l'école sociale populaire au Québec (1911-1930)"

"The origins of the dialetic of Marx's idea of revolution" «Spécialisation internationale et branches de la production:

le cas de Singapour"

"Crise et politique de développement économique au Québec: le cas de la Baie James"

"La stratégie phosphatière du Maroc"

«Kissinger et le problème de la conversion de l'URSS en une puissance conventionnelle "

«Décentralisation et projet éducatif: analyse de la nouvelle politique de l'éducation au Québec"

"L'aide alimentaire en République Islamique de Mauritanie de 1979 à 1983"

«La crise du Welfare State et ses conséquences sur la pratique en travail social dans le secteur public et para-public au Québec."

«Pouvoirs intégrés à l'État. Pouvoirs parallèles à l'État. Les bases sociales du pouvoir politique central au Sénégal " "Le travail à temps partagé au Canada "

"La conception du politique chez Claude Lefort"

«Les relations comparées de la France et du Canada avec l'Afrique: l'exemple de la République centrafricaine" «Science politique, scientificité et pouvoir: pour un appareil critique"

«La pensée politique d'Hannah Arend: essai d'interprétation" «Monde contemporain, autonomie et révolution rétrospective et commentaire de l'œuvre de Cornelius Catoriadis»
Université d'Ottawa

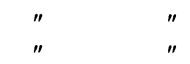

Université du Québec à Montréal
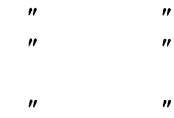

"

"
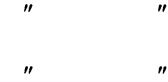

"

$"$

" 
Asselin, Hélène

Berthiaume, Jean-Marie

Charbonneau, Paul-M.

Chouinard, Jean-Yves

Côté, Pauline

Coutts, Douglas

Daudelin, Jean

Desjardins, Luc

Dioury, Mohamed

Gauron, Michel

Gerlier-Forest, Pierre Godin, Benoît

Goyette, Line
"Les engagements politiques d'Emmanuel Mounier: introduction à l'étude des rapports entre morale et politique"

"Réflexions préliminaires sur la genèse du procès idéologique"

«Étude de stratégie d'un groupe minoritaire: le cas de l'enseignement secondaire français en Ontario

«Esquisse d'un modèle prospectif pour un processus d'accession à la souveraineté: le cas québécois"

«De la dévotion au pouvoir: les femmes dans le renouveau charismatique"

"Le développement politique, les relations transnationales et le peuple autochtone de l'Arctique: la conférence inuit circumpolaire"

"La régulation étatique: le cas du Nicaragua sandiniste»

"Nationalisme et régionalisme: une analyse spatiale de la cohésion nationale en Acadie du Nouveau-Brunswick *

«Économie politique du Sénégal (1860-1980). Essai d'interprétation"

«La stratégie générale de troisième option dans la politique étrangère canadienne: l'Amérique latine comme région cible privilégiée d'application"

"Pour une approche générative des partis politiques»

"La modélisation des phénomènes électoraux: pour une théorie du comportement rationnel des électeurs"

«Le travail domestique : entre l'objectivité et la subjectivité (le cas des ménagères au Québec)»
Université Laval

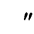

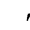

"

"

Université Laval

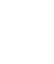

\begin{tabular}{|c|c|}
\hline " & " \\
\hline " & $"$ \\
\hline$"$ & " \\
\hline " & $"$ \\
\hline " & $"$ \\
\hline " & " \\
\hline " & "ום \\
\hline
\end{tabular}


Kampiré, Marie-Thérèse

Nicoloff, Olivier

Ostry, Adam

Pierre-Jérôme, Frantz

Thomassin, Philipe

Charlton, Mark William

Baird, Edison Alva

Bartley, Allan

Chernin, Mark D.

Desrochers, Sylvain

Duquette, Michel

Éthier, Jean
«Analyse des contraintes relatives à l'octroi de l'aide extérieure au Rwanda"

"L'arme alimentaire. Essai sur l'utilisation des échanges céréaliers à des fins politiques "

«Fédéralisme, nationalisme et social-démocratie: le nouveau parti démocratique et le Québec»

"Le pouvoir des entreprises multinationales sur l'État: le cas du cuivre au Chili»

"Au-delà de la dichotomie religieux-politique, trois types idéaux de régulation"

"The management of canadian food aid policy: a study in donor structure and aid strategy"

"Hungary and South Korea : a comparative analysis of their export performances from a political economy perspective, 1969-1980"

"The magic solution: the cross-media ownership direction" "Decision-making in crisis: Canada's entry into world war II"

«La participation canadienne à l'Otan (1945-1980): une analyse de la pensée stratégique canadienne»

«Politiques de l'énergie au Brésil et crise de la triple alliance avec le programme Pro-Alcool»

«La répartition de la richesse et des revenus: performances gouvernementales du Nouveau Parti Démocratique de la Colombie Britannique et du Parti Québécois»
Université Laval

"

Université Mc Gill

" 
Lévesque, Gérard

Levy, Roger Peter

Ruvinsky, Ilene G.

Saldanha, Ashley

Thomas, Brownlee
«Transformation of the political leadership of the German Democratic Republic (1945-1981): an analysis of the social, professional and political backgrounds of the new full members of the Central Committee of the Socialist Unity Party"

«Reshaping the nationalist appeal: public opinion, party strategy and the Scottish Nationalist Party

"The role of the "Gazette" in linguistic group relations in Quebec: a contextual approach"

"State power and social classes in Tanzania"

«The role of communication in economic development "
Université McGill 